\title{
Evaluation of Toxoplasma gondii as a live vaccine vector in susceptible and resistant hosts
}

\author{
Jun Zou', Xiao-Xi Huang ${ }^{1}$, Guang-Wen Yin', Ye Ding ${ }^{2}$, Xian-Yong Liu', Heng Wang ${ }^{3}$, Qi-Jun Chen ${ }^{4}$ and Xun Suo ${ }^{1 *}$
}

\begin{abstract}
Background: Toxoplasma gondii has been shown to trigger strong cellular immune responses to heterologous antigens expressed by the parasite in the inbred mouse model [1]. We studied the immune response induced by T. gondii as an effective vaccine vector in chickens and rabbits.
\end{abstract}

Results: T. gondii RH strain was engineered to express the yellow fluorescent protein (YFP) in the cytoplasm. A subcutaneous injection of the transgenic T. gondii YFP in chickens afforded partial protection against the infection of transgenic E. tenella YFP. T. gondii YFP induced low levels of antibodies to YFP in chickens, suggesting that YFP specific cellular immune response was probably responsible for the protective immunity against $E$. tenella YFP infection. The measurement of T-cell response and IFN- $\gamma$ production further confirmed that YFP specific Th1 mediated immune response was induced by T. gondii YFP in immunized chickens. The transgenic T. gondii stimulated significantly higher YFP specific IgG titers in rabbits than in chickens, suggesting greater immunogenicity in a T. gondii susceptible species than in a resistant species. Priming with T. gondii YFP and boosting with the recombinant YFP can induce a strong anti-YFP antibody response in both animal species.

Conclusions: Our findings suggest that $T$. gondii can be used as an effective vaccine vector and future research should focus on exploring avirulent no cyst-forming strains of $T$. gondii as a live vaccine vector in animals.

\section{Background}

A variety of viruses and bacteria have been used successfully as live vaccine vectors [2-6]. The antigen delivering efficiency and the type of immune response of live vaccine vectors depends on their replication at infected sites and in target cells [7]. An effective live vaccine vector should have the capacity to infect a wide range of target cells with high efficiency and present effectively heterologous antigens to $\mathrm{T}$ cells. In addition, a live vaccine vector should also satisfy the requirement of safety and the ease of transfection of foreign DNA into the vector [8].

Toxoplasma gondii is an obligate intracellular parasite. It can infect any nucleated cells of warm-blood vertebrates [9-12] and induce strong humoral, mucosal and cellular immune responses, making it an attractive system for delivering heterologous antigens [9]. Avirulent strains of $T$. gondii have been tested to immunize

\footnotetext{
* Correspondence: suoxun@cau.edu.cn

'National Animal Protozoa Laboratory, College of Veterinary Medicine, China Agricultural University, Beijing, 100193, China

Full list of author information is available at the end of the article
}

livestock and studied in experimental animals to prevent congenital toxoplasmosis [13]. A commercial live S48 strain vaccine (Ovilis. Toxovax ${ }^{\circledR}$ ) for veterinary use has already been approved in some countries [14-16]. Because of the strong immunogenicity, availability of avirulent strains and the ease of genetically engineering stable parasite lines, $T$. gondii has the potential to be explored as a live vaccine vector for bacterial, viral and parasite pathogens [17].

Studies on the immune response to $T$. gondii infection have been conducted extensively in the mouse $[1,18,19]$. Green fluorescent protein (GFP) has been extensively utilized as the reporter protein in genetic manipulation [20-22], and it was also used as a model antigen to study the antigen delivery to target the specific immune response pathway [23]. We posed the following questions: (-) Could foreign antigens expressed by $T$. gondii stimulate antigen-specific protective immune responses in chickens; $(-)$ whether there is any difference in antigen specific immune responses induced by transgenic $T$. gondii in chickens, which are naturally resistant to $T$.
C Biomed Central 
gondii infection, and rabbits, which are susceptible to $T$. gondii infection.

In this study, we developed a transgenic $T$. gondii that expressed the yellow fluorescent protein (YFP), a yellow version of GFP [24], as a model antigen. We firstly demonstrated that the transgenic $T$. gondii YFP elicited YFP-specific immune responses that conferred partial protection against a challenge with YFP-expressing $E$. tenella. We also showed that immunization with transgenic T. gondii YFP induced greater YFP-specific humoral immune responses in rabbits than in chickens. Our data have obvious implications on the utilization of T. gondii or other apicomplexa protozoa as a live vaccine vector. A commercial live vaccine strain S48 or avirulent no cyst-forming strains of $T$. gondii need to be used to explore T. gondii as a live vaccine vector in animals in the future study.

\section{Materials and methods}

\section{Parasite}

The wild type RH strain of $T$. gondii and its stably transfected line were maintained by serial passages in African green monkey kidney (VERO) (Shanghai Institutes For Biological sciences, CAS) cells in DMEM supplemented with FBS $(10 \% \mathrm{v} / \mathrm{v})$, penicillin $\left(200 \mathrm{U} \mathrm{ml}^{-1}\right)$ and streptomycin $\left(20 \mathrm{mg} \mathrm{ml}^{-1}\right)$ in a humidified atmosphere of $5 \% \mathrm{CO}_{2}$ at $37^{\circ} \mathrm{C}$. Stable YFP-transfected Eimeria tenella (E. tenella YFP) was constructed, maintained and propagated in coccidia-free 4-day-old AA broilers [25], briefly YFP expression vector was transfected into the wild type E. tenella sporozoites, and the transfected sporozoites were inoculated into chickens. At 6-9 days post-infection, oocysts were collected from feces of chickens according to procedures described previously [26]. The YFP positive oocysts were sorted by a MoFloTM cell sorter (Dako Cytomation, Denmark) four times until the percentage of fluorescent oocysts reached $90 \%$.

\section{Plasmid construct}

The pTgmicYFP plasmid was constructed from the pTgsagYFP, which was previously constructed in our laboratory [27]. The sag1 promoter of $T$. gondii was replaced by the $T$. gondi microneme 2 (MIC2) promoter $(1.48 \mathrm{~kb})$ before the insertion of the YFP reporter gene within the 5' sequence of MIC2 and 3' sequence of SAG1 of $T$. gondii (Figure 1A).

\section{Transgenic $T$. gondii}

The RH strain T. gondii tachyzoites were propagated, harvested and purified according to established protocols [28]. Freshly purified tachyzoites were suspended in
A

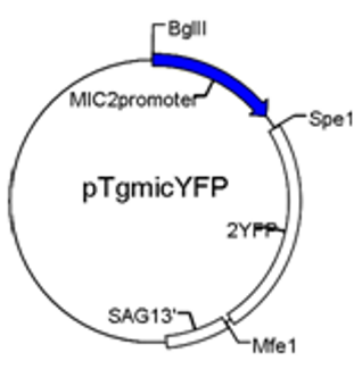

C

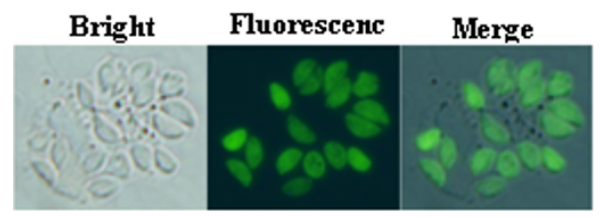

B

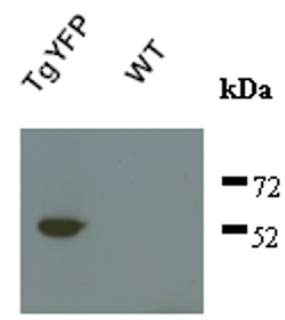

D

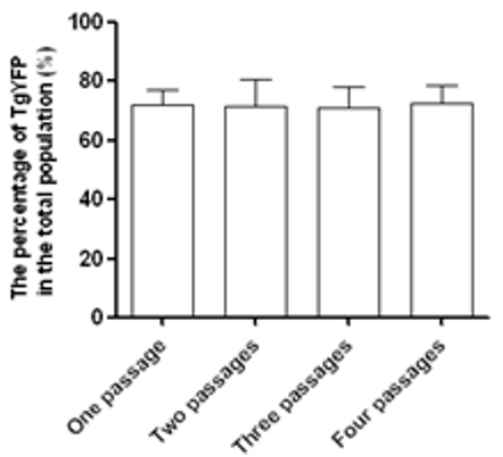

Figure 1 Expression of yellow fluorescent protein (YFP) by T. gondii transfected with the pTgmicYFP plasmid. A, Plasmid map of pTgmicYFP. B, Western blot of YFP expressed by the transgenic T. gondii YFP (TgYFP) and wild type T. gondii (WT) using the rabbit anti-GFP antisera and a sheep anti-rabbit lgG HRP-conjugate. C, T. gondii YFP in murine macrophages observed by fluorescence microscopy, confirming the location of YFP by T. gondii YFP. D, Fitness of T. gondii YFP and wild type T. gondii at a ratio of 7:3 in mice. 
the complete cytomix buffer to a final concentration of $5 \times 10^{7} \mathrm{ml}^{-1}$. The pTgmicYFP plasmid was linearized with the restriction endonuclease BglII and transfected to the parasites by electroporation [29]. Fifty-150 $\mu$ l linearized pTgmicYFP, $100 \mathrm{U}$ BglII and $1 \times 10^{7}$ tachyzoites in a $4 \mathrm{~mm}$ cuvette were subjected to electroporation (Gene Pulser II ${ }^{\mathrm{TM}}$, Bio-Rad, USA) at a peak voltage of 2 $\mathrm{kV}$ and a capacitance of $25 \mu \mathrm{F}$. After electroporation, the parasites were left undisturbed at room temperature for $20 \mathrm{~min}$ and then inoculated onto confluent MDBK or VERO cells (Shanghai Institutes For Biological sciences, CAS) in the modified DMEM medium.

The transfected tachyzoites were released and sorted by a MoFlo ${ }^{\mathrm{TM}}$ cell sorter (Dako Cytomation, Denmark), and the sorted tachyzoites were cultured again in MDBK or VERO cells. After about five passage-sorting cycles, the transgenic line, named as $T$. gondii YFP was cloned by limiting dilution in 96-well plates [28].

YFP expression in the transfected tachyzoites was verified by Western blotting. The transgenic and wild parasites were harvested from VERO cells, lysed in SDS sample buffer, and boiled for $10 \mathrm{~min}$. The YFP protein was identified by Western blotting using rabbit antiGFP antisera (Proteintech, China), goat anti-rabbit IgG conjugated to alkaline phosphatase (Proteintech, China) and chemiluminescent detection (CWBIO, China).

YFP expression by the transgenic line in vivo was studied in mice. The transgenic T. gondii YFP was propogated in Balb/c mice by i.p. inoculation with $1 \times 10^{4}$ tachyzoites per animal. Replicating $T$. gondii YFP tachyzoites in murine macrophages were harvested from the peritoneal cavity with $4 \mathrm{ml}$ of sterile saline 3 days after inoculation. Parasites in intact macrophages were examined by fluorescence microscopy with $488 \mathrm{~nm}$ excitation and $508 \mathrm{~nm}$ emission filters.

To determine the fitness of $T$. gondii YFP, the transgenic and wild type $T$. gondii at a ratio of $7: 3$ were inoculated i.p. to mice at $10^{4}$ /animal. The proportion of T. gondii YFP, which were harvested from the peritoneal cavity 3-5 days after inoculation, was measured by FACS after each passage in mice.

\section{Tachyzoite antigens preparation and expression of YFP proteins in bacteria}

A crude extract of proteins was obtained by repeated freezing and thawing of $2 \times 10^{8} \mathrm{RH}$ tachyzoites in liquid nitrogen. The lysates were centrifuged at $5000 \times \mathrm{g}$ for $20 \mathrm{~min}$ at $4^{\circ} \mathrm{C}$ and the supernatants were collected. The YFP gene was cloned from the pTgtubNP-YFP/sagCAT vector [30]. The cloned gene was inserted into the $\mathrm{pET}$ 21 a vector (Novatech, France). The resulting plasmid was transformed to E. coli BL21 (DE3) cells (Transgen Company, China) with ampicillin selection. The recombinants were harvested after $6 \mathrm{~h}$ of induction with IPTG
(Isopropyl $\beta$-D-1-Thiogalactopyranoside). The YFP protein was purified using a His bind buffer kit (Novatech, France).

\section{Animals and immunization}

AA broiler chickens were separately caged in an air-conditioned room. The 15 -day-old chickens $(n=10)$ were immunized s.c. or i.m. with the transgenic or wild type T. gondii $\left(5 \times 10^{6} /\right.$ chicken $)$ in complete cytomix buffer (CCB), $160 \mu \mathrm{g}$ recombinant YFP emulsified in Freund complete adjuvant (FCA) or CCB. Female New Zealand white rabbits (90 days of age) were inoculated with $1 \times$ $10^{7}$ parasites. The immunized or non-immunized chickens and rabbits were boosted i.m. with $160 \mu \mathrm{g}$ (chicken) or $500 \mu \mathrm{g}$ (rabbit) YFP emulsified with FCA. The booster dose was administered 20 (chicken) or 35 (rabbit) days after the primary immunization. Serum anti-YFP antibodies were determined by ELISA 10 days after the primary immunization or the booster immunization.

Additional groups of 10 day old white Leghorn chickens $(n=10)$ were immunized s.c. twice with the transgenic or wild type $T$. gondii tachyzoites or160 $\mu \mathrm{g}$ recombinant YFP emulsified in Freund complete adjuvant (FCA) or complete cytomix buffer (un-immunized control) at a dose interval of 15 days. The primary immunization tachyzoites was $5 \times 10^{6}$, and the booster dose was $1 \times 10^{7}$. All chickens were challenged orally with transgenic E. tenella YFP oocysts $\left(1 \times 10^{3}\right) 15$ days after the booster immunization dose. The immune protection of T. gondii YFP against E. tenella YFP, which carry the same model antigen, was assessed by fecal oocyst excretion and cecum lesions 9 days after the challenge.

\section{Determination of antibody titers}

Serum antigen specific IgG of the immunized chickens or rabbits was measured by ELISA. A 96-well microtiter plates were coated with the recombinant YFP harvested from E. coli BL21 bacteria (described above) at $2 \mu \mathrm{g} / \mathrm{ml}$ or tachyzoite antigens at $5 \mu \mathrm{g} / \mathrm{ml}$ in $0.05 \mathrm{M}$ bicarbonate buffer ( $\mathrm{pH}$ 9.6) overnight at $4^{\circ} \mathrm{C}$, and blocked for $2 \mathrm{~h}$ at $37^{\circ} \mathrm{C}$ with $5 \%$ milk powder (Difco ${ }^{\mathrm{TM}}$ skim milk, BD) in PBST(PBS containing 0.05\% Tween 20)before washing with PBST. Serially diluted serum samples were added and incubated for $1 \mathrm{~h}$ at $37^{\circ} \mathrm{C}$. Antigen-specific antibodies were detected with HRP-labeled anti-chicken IgG with 1:1000 dilution or anti-rabbit IgG with 1:5000 dilution in $2 \%$ milk-PBST for $1 \mathrm{~h}$ at $37^{\circ} \mathrm{C}$. The ELISA was developed with $100 \mu \mathrm{l} /$ well of $10 \mathrm{mg} / \mathrm{ml} \mathrm{TMB}$ solution in $0.025 \mathrm{M}$ phosphate-citrate buffer (Sigma). The reaction was stopped by $0.2 \mathrm{M} \mathrm{H}_{2} \mathrm{SO}_{4}$ and the optical density (OD) at 450 and $630 \mathrm{~nm}$ was measured by a plate reader (Bio-Rad, CA, USA). The serum antibody titer was defined as the highest dilution that gave a test/naive serum OD ratio of 2.1 or higher. 


\section{Real-time RT-PCR}

Three chickens of each group were sacrificed on the 12th day after immunization. Single-cell suspensions derived from spleen in RPMI-1640 plus 10\% FCS were prepared and loaded onto 6-well cell cultured plates $\left(10^{7}\right.$ cells per well). The cells were incubated at $41^{\circ} \mathrm{C}$ in a $5 \% \mathrm{CO}_{2}$ incubator. The cells were stimulated for $16 \mathrm{~h}$ with rYFP $(5 \mu \mathrm{g} / \mathrm{ml})$. Total RNA was extracted with Tri$\mathrm{zol}^{\circledR}$ (Invitrogen) and then transcripted using High Capacity cDNA Reverse Transcription Kit (Applied Biosystems). The primer pairs used for analysis of cDNA were showed in Table 1. Quantitative real-time PCR was performed on the 7500 Real Time PCR System (Applied Biosystems) with a program of $50^{\circ} \mathrm{C}$ for $2 \mathrm{~min}, 95^{\circ} \mathrm{C}$ for $10 \mathrm{~min}$ and 40 cycles of $95^{\circ} \mathrm{C}$ for $15 \mathrm{~s} ; 60^{\circ} \mathrm{C}$ for $1 \mathrm{~min}$. For each sample, template copy numbers were internally normalized with their respective input control. Relative expression was calculated as the ratio of template copy numbers of a sample relative to the naive control after normalizing to their respective isotype control Actin.

\section{Histological examination}

The cecum of the challenged chickens was collected and fixed in $2.5 \%(\mathrm{v} / \mathrm{v})$ glutaraldehyde-polyoxymethylene solution immediately after euthanization. The tissue samples were dehydrated and embedded in paraffin wax. Serial paraffin sections ( $4 \mathrm{um}$ ) were obtained and incubated at $37^{\circ} \mathrm{C}$ for at least $12 \mathrm{~h}$. Paraffin was removed by three consecutive washings in xylol for $5 \mathrm{~min}$ each, and then hydrated with in 100, 95, 80, 70 and 50\% alcohol and then deionized water. The histological paraffin sections were stained with Hematoxylin \& Eosin (HE) and examined under a light microscope.

\section{Statistical analysis}

The data were analyzed using the one-sided student's ttest. A P value less than 0.05 or 0.01 was considered significant.

\section{Results}

Transgenic $T$. gondii expressing the heterologous protein YFP

We engineered transgenic $T$. gondii expressing YFP as a model antigen by transfection of $T$. gondii $\mathrm{RH}$ with the plasmid pTgmicYFP containing a strong MIC2

Table 1 Primer sequences used in Real-time RT-PCR

\begin{tabular}{ccc}
\hline Sequence no & Gene name & \multicolumn{1}{c}{ Primer sequence } \\
\hline 1 & IFN- $\gamma$ & F-CGCACATCAAACACATATCTG \\
& & R-GATTCTCAAGTCGTCATCGG \\
2 & IL-4 & F-AGGCAACACTACTCAATGG \\
& & R-GCTAGTTGGTGAAGAAGGT \\
3 & Actin & F-CCACACTITCTACAATGAGCTG \\
& & R-GGTCTCAAACATGATCTGTGTC \\
\hline
\end{tabular}

promoter (Figure 1A). A stable transgenic line was cloned after several passages in mice and FACS sorting and limiting dilution. The exotic YFP protein expression was confirmed by western blotting. The transgenic $T$. gondii produced an expected band of $54.6 \mathrm{kDa}$ protein recognized by anti-GFP antibodies, while no positive band was detected for the wild type $T$. gondii (Figure 1B). Fluorescence microscopy showed that $T$. gondii YFP expressed abundant YFP protein which was located in the cytoplasm of the parasites (Figure 1C). The transgenic $T$. gondii was co-passaged with the wild type $T$. gondii in mice and the proportion of the two lines remained constant after 4 passages (Figure 1D); this indicated that the fitness of the transgenic parasites was not altered after the integration of the exotic DNA into the parasite genome.

\section{Immunization with transgenic $T$. gondii YFP conferred} partial protection against challenge with $E$. tenella YFP It has been shown that immunization of inbred mice with transgenic $T$. gondii expressing a foreign antigen of microorganisms can provide highly effective priming for CD8 T cell-dependent protective immunity against the microorganisms $[1,18]$. The YFP specific protective immune response was studied in chickens by immunization with $T$. gondii YFP followed by challenging with transgenic $E$. tenella YFP expressed in the cytoplasm (Figure 2A). Immunization with $T$. gondii YFP provided partial protection against $E$. tenella expressing the same antigen, YFP. Fecal oocyst output was significantly lower in the $T$. gondii YFP-immunized chickens than the wild type $T$. gondiiimmunized or un-immunized chickens. The latter two groups yielded comparable number of oocysts. Compared with the rYFP immunized group, there was a decrease in fecal oocyst output in the T. gondii YFP-immunized chickens; although the small decrease in fecal oocyst output was not statistically significant (Figure 2B).

Histological examination of the cecum showed shedding of mucosal cells and inflammatory cell infiltration of the lamina propria in all groups, but the lesions in the transgenic $T$ gondii group were less severe than the wild type and naïve groups and comparable to the rYFP immunized group (Figure $2 \mathrm{C}$ ). These data suggest that the transgenic T. gondii YFP can elicit YFP specific immune responses in chickens. Previous studies showed that poultry are protected from coccidiosis mainly by cellular immunity $[31,32]$. The protection against E. tenella YFP infection in chickens observed in this study indicated that $T$. gondii YFP induced YFP specific cellular immunity.

\section{Humoral immune responses of chickens and rabbits to the transgenic $T$. gondii}

Here we investigated YFP specific humoral immune response in chickens and rabbits immunized with the 
A

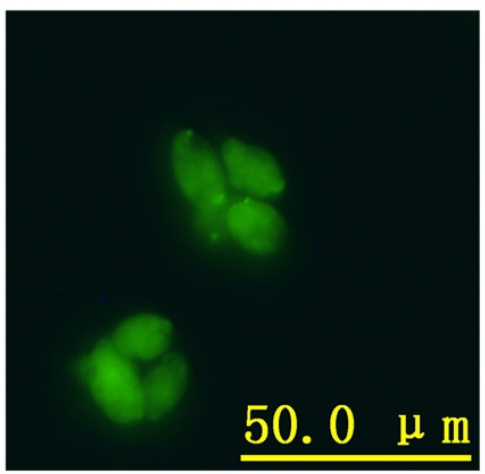

C - /EiYFP
Wild-Tg /EiYFP
B

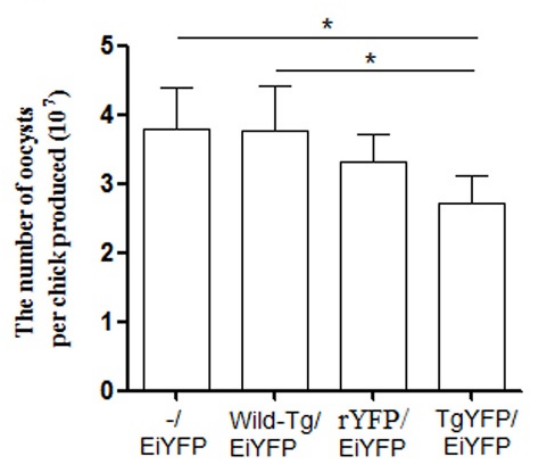

rYFP /EIYFP
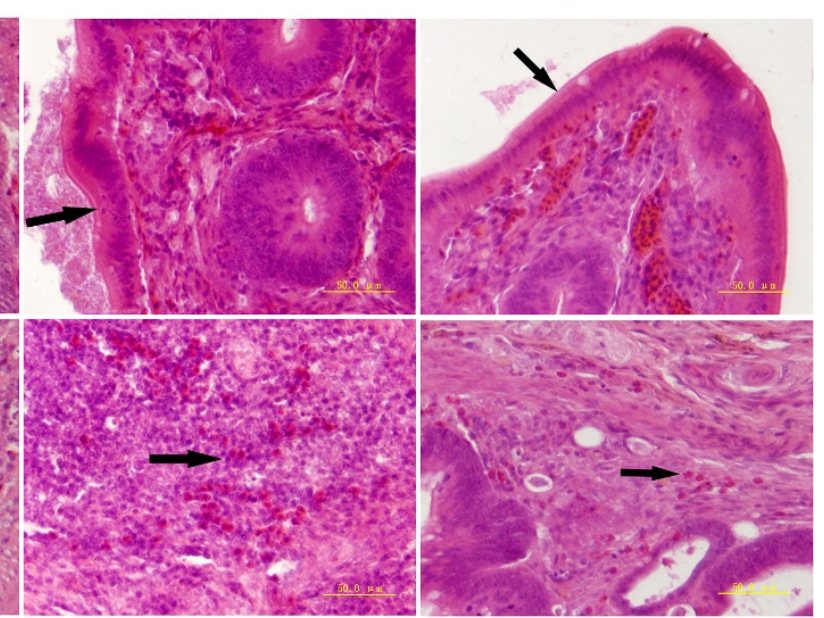

Figure 2 The partial protection against challenge with Eimeria tenella YFP (EiYFP) in chickens which were immunized with T. gondii. Leghorn chickens immunized s.c. with two doses of T gondii YFP (TgYFP) or wild type RH strain $\left(5 \times 10^{6}\right.$ for the initial immunization and $10^{7}$ for the booster dose) or $160 \mu \mathrm{g}$ recombinant YFP emulsified in Freund complete adjuvant (FCA). The immunized chickens were challenged with $10^{3}$ transgenic E. tenella YFP 15 days after the booster immunization. A. Fluorescence images of transgenic E. tenella YFP oocysts. B. Fecal oocyst counts in chickens immunized with T. gondii YFP (TgYFP) or wild type T. gondii tachyzoites (Wild-Tg) or recombinant YFP emulsified in FCA (rYFP) or complete cytomix buffer (un-immunized control) and challenged with the transgenic E. tenella (EiYFP). C. Histopathology of the cecum from chickens described above in Figure B. $C_{1}$ Shedding of mucosal cells (arrow); $C_{2}$ Inflammatory cells infiltration of lamina propria (arrow).

transgenic T. gondii. AA broiler chickens were immunized s.c. or i.m. with $5 \times 10^{6}$ transgenic or wild type $T$. gondii tachyzoites (WT), $160 \mu \mathrm{g}$ rYFP emulsified in FCA or CCB. Chickens primed with $T$. gondii YFP developed YFP-specific IgG, and the levels of anti-YFP IgG were significantly higher than those in chickens primed with wild type $T$. gondii or CCB (Figure 3A). Serum antibody titers in chickens injected i.m. with the transgenic parasites were comparable with the titers in chickens immunized by the s.c. route (Figure 3B). However, YFP specific antibody titers in chickens immunized with the transgenic parasites were markedly lower than those in chickens immunized with the rYFP protein (Figure $3 \mathrm{C}$ ). Next, we investigated the humoral immune response in a susceptible animal model, the rabbit, to the transgenic T. gondii. Rabbits were immunized s.c. with $1 \times 10^{7} T$. gondii YFP or WT strain, and serum antibodies to YFP and tachyzoite antigens were measured. A subcutaneous injection of the transgenic parasite elicited high levels of YFP specific antibodies, The IgG titers peaked on day 25 post immunization (Figure 3D). Compared with YFP specific antibodies, the host produced higher level of IgG to tachyzoite antigens (Figure 3D).

Prior immunization with $T$. gondii YFP had no impact on humoral immune response to rYFP in either animal species. Similar serum YFP specific antibody titers were measured in chickens and rabbits injected with rYFP with or without prior immunization with $T$. gondii YFP (Figure 4). The above findings suggest that a subunit vaccine may be utilized to induce high level of humoral immune response in some animal species to make up for the low IgG titre induced by transgenic $T$. gondii. 
B
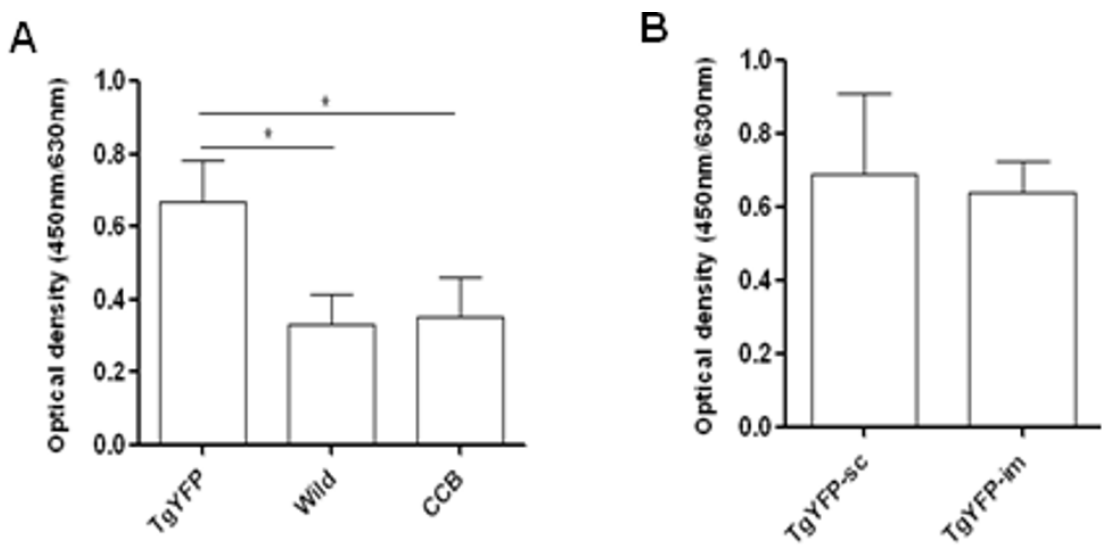

C

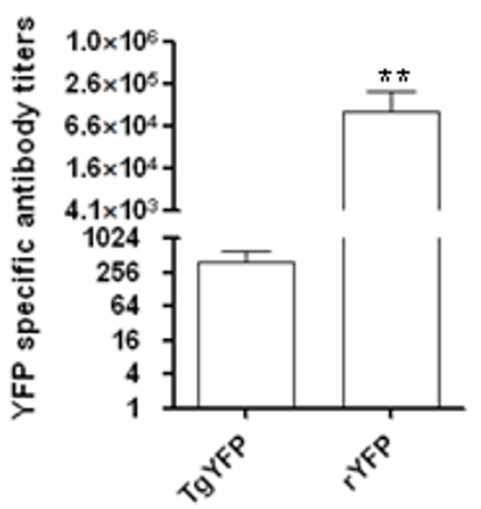

D

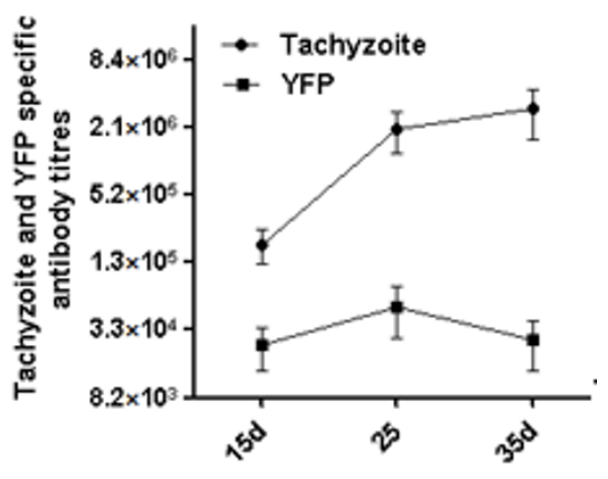

Figure 3 Priming of AA broiler chickens and rabbits with T. gondii YFP (TgYFP) elicited antigen specific antibody responses. A, YFP specific lgG in chicken sera (1:25) 10 days after immunization by s.c. injection of $5 \times 10^{6}$ transgenic (TgYFP), wild type (WT) T. gondii tachyzoites or complete cytomix buffer (CCB). B, YFP specific lgG (1:25) 10 days after immunization by s.c. or i.m. injection. C, Serum YFP specific antibody titers in chickens 10 days after a s.c. injection of transgenic T. gondii tachyzoites (TgYFP) or i.m. injection of recombinant YFP (rYFP) protein expressed in $E$. coli. D, Kinetics of serum antibodies to YFP and tachyzoite antigens in rabbits immunized with the transgenic T. gondii. ${ }^{*} P<0.05,{ }^{* *} P<0.01$.

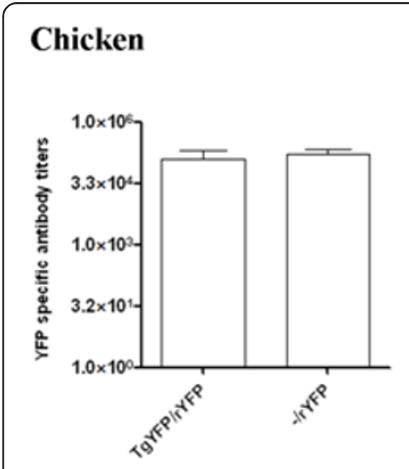

\section{Rabbit}

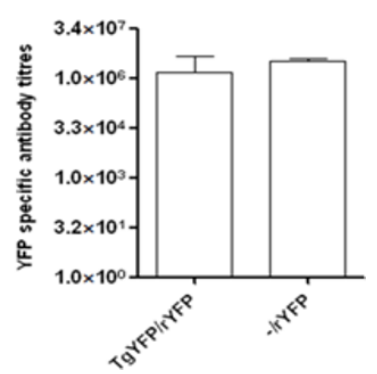

Figure 4 Serum YFP specific antibody titers in chickens and rabbits immunized with $T$. gondii YFP and/or rYFP protein. Chickens and rabbits were primed s.c. with $5 \times 10^{6}$ and $1 \times 10^{7} \mathrm{~T}$. gondii YFP tachyzoites, respectively. The primed chickens were boosted i.m. 20 days later with $160 \mu \mathrm{g}$ of rYFP emulsified in Freund complete adjuvant, and the primed rabbits were boosted i.m. 35 days later with $500 \mu \mathrm{g}$ of recombinant YFP emulsified in Freund complete adjuvant. Serum YFP specific IgG titers were measured 10 days after the booster immunization.

\section{Transgenic $T$. gondii elicited YFP-specific cytokines} expression

To further determine the YFP specific immune response induced by transgenic $T$. gondii YFP, we examined the level of IFN-g (Th1 type) and IL-4 (Th2 type) production by YFP specific $\mathrm{T}$ cells using Real-time RT-PCR. Single cell suspensions of lymphocytes were prepared from the spleen of the immunized chickens on day 12 after immunization, and restimulated in culture with rYFP. The results showed that the lymphocytes from transgenic $T$. gondii YFP immunized chickens produced significantly higher level of IFN- $\gamma$ compared with wild type (WT) tachyzoites immunized group, while analysis of the IL-4 transcription revealed that there was no significant difference between the transgenic group (TgYFP) and the WT group (Figure 5).

\section{Discussion}

Transgenic organisms expressing model antigens (e.g. ovalbumin, $\beta$-galactosidase) have been conveniently used 


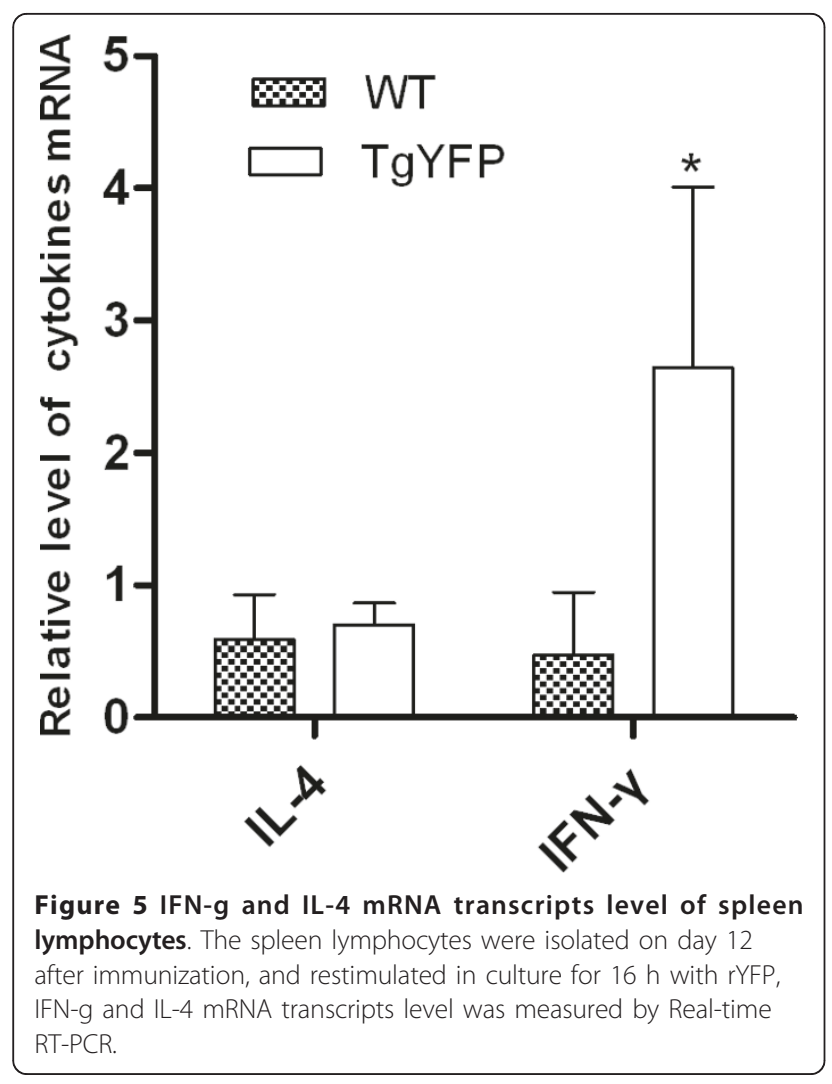

to search for effective vaccine vectors. Protective effects of the model antigen specific immune response are evaluated by challenging the host with a different virus or bacteria strain or species expressing the same antigen [33]. Although some studies have already begun to explore the apicomplexan parasites as live vaccine vectors [34-38], there had been no studies to use transgenic parasites to express model antigen and induced immunity against infection by other transgenic Apicomplexa parasites, which have more complex life cycles and express more proteins than viruses and bacteria. Although both Eimeria tenella and Toxoplasma gondii are intracellular Apicomplexa parasites, there are significant differences in genome, proteome and life cycle $[39,40]$. Our study showed that the transgenic $T$. gondii expressing YFP induced immunity that partially protected chickens from the challenge with the transgenic E. tenella also expressing YFP. Therefore this study paved the way for the application of a heterologous challenge system to apicomplexa parasites.

Variations in susceptibility of different animal species to $T$. gondii infection are well known. The resistance is probably attributable to innate immunity or a rapid and strong immune response before the parasite can even disseminate. Chickens inoculated with $T$. gondii do not show any clinical symptoms [41], while rabbits are highly susceptible to T. gondii infection [42]. Both chickens and rabbits were chosen to evaluate the humoral immunity induced by the transgenic $T$. gondii. We observed that the YFP specific humoral immune response in the rabbit was significantly higher than that in the chicken. This implied that the susceptibility was one important determinant of the strength of immune responses. It was reported that six of seven $T$. gondii isolates from chickens were avirulent in mice, suggesting that the chicken was possibly more susceptible to avirulent $T$. gondii infection. Therefore, the avirulent $T$. gondii strain may be an effective vaccine vector for chickens.

It was reported that subunit vaccines predominantly induced high level neutralizing antibodies. To enhance the humoral immune response induced by the transgenic $T$. gondii in resistant animals, chickens immunized with $T$. gondii YFP were boosted with the YFP protein. The combined immunization regimen induced a high level humoral response in chickens compared to the weak humoral immunity induced by the transgenic parasite alone. Chicken whether or not they are vaccinated with $T$. gondii YFP prior to injection with rYFP produce the equivalent titers of anti-YFP antibody, this may be due to the superior ability of rYFP emulsified in FCA to induce high antibody titer which makes the effect of a boost is not visible. For the elicitation of strong cellular and humoral immune responses by transgenic $T$. gondii, the immunization with a combination of trangesnic parasites-based vaccine and a subunit vaccine should be considered.

Some intracellular pathogens have the coding capacity to produce distinct proteins that are sorted into different intracellular compartments $[43,44]$. Studies have shown that only a few proteins of $T$. gondii can stimulate CD8 T cell-dependent immunity in mice model [44,45]. Secreted, not cytoplasmic, antigens of T. gondii primed IFN- $\gamma$ expressing CD8 $\mathrm{T}$ cells in mice which was susceptible to T. gondii infection [46]. Chicks, which were resistant to $T$. gondii infection, have many immunological mechanisms in common with mammals but have evolved distinct immunity to pathogens [47]. Here we showed that cytoplasm-localized YFP expressed by $T$. gondii can produce protective immune response in chicks that conferred partial protection against challenge with E. tenella YFP. To investigate whether this partial protection was related to the cytoplasm localization of YFP, a transgenic T. gondii line which secreted YFP need to be constructed in the future study to investigate the influences of antigen compartmentalization on the immune response in chickens.

\section{Conclusions}

In conclusion, our study demonstrated the feasibility of using $T$. gondii as a delivery system to induce antigens specific protective immunity against the heterologous 
pathogen. The recombinant $T$. gondii-based vaccine priming and subunit vaccine boosting approach would be more effective than immunization with the transfected parasites alone in some animal species. The optimizations of $T$. gondii as a live vaccine vector to enhance the immune response need to be conducted in the future study.

\section{Acknowledgements}

This study was supported by Graduate Research and Innovation Program (Project numbers: 15059206) and the National S \& T Major Program (Grant No. 2008ZX10004-011). We declare that the experiments complied with the current laws of China where they were performed.

\section{Author details}

'National Animal Protozoa Laboratory, College of Veterinary Medicine, China Agricultural University, Beijing, 100193, China. ${ }^{2}$ Department of Veterinary Pathology, College of Veterinary Medicine, China Agricultural University, Beijing, 100193, China. ${ }^{3}$ Department of Etiology, Molecular Parasitology Laboratory, Institute of Basic Medical Sciences, Chinese Academy of Medical Sciences and Peking Union Medical College, Beijing 100005, China. ${ }^{4}$ Institute of Pathogen Biology, Chinese Academy of Medical Sciences, Dong Dan San Tiao 9, Beijing 100730, China.

\section{Authors' contributions}

JZ, XXH, GWY, YD all participated in collecting the results presented here: HW and QJC contributed to the revision of the manuscript; XYL and XS supervised the study implementation and revised the manuscript. All authors read and approved the final manuscript.

\section{Authors' information}

National Animal Protozoa Laboratory ${ }^{a}$, and Department of Veterinary Pathology ${ }^{\mathrm{b}}$, College of Veterinary Medicine, China Agricultural University, Beijing, 100193, China. ' Department of Etiology, Molecular Parasitology Laboratory, Institute of Basic Medical Sciences, Chinese Academy of Medical Sciences and Peking Union Medical College, Beijing 100005, China.

d Institute of Pathogen Biology, Chinese Academy of Medical Sciences, Dong Dan San Tiao 9, Beijing 100730, China.

\section{Competing interests}

The authors declare that they have no competing interests.

Received: 6 April 2011 Accepted: 28 August 2011

Published: 28 August 2011

\section{References}

1. Charest H, Sedegah M, Yap GS, Gazzinelli RT, Caspar P, Hoffman SL, Sher A: Recombinant attenuated Toxoplasma gondii expressing the Plasmodium yoelii circumsporozoite protein provides highly effective priming for CD8+ T cell-dependent protective immunity against malaria. J Immunol 2000, 165:2084-2092

2. Bukreyev A, Skiadopoulos MH, Murphy BR, Collins PL: Nonsegmented negative-strand viruses as vaccine vectors. J Virol 2006, 80:10293-10306.

3. Zhang $X L$, Jeza VT, Pan Q: Salmonella typhi: from a human pathogen to a vaccine vector. Cell Mol Immunol 2008, 5:91-97.

4. Konjufca V, Wanda SY, Jenkins MC, Curtiss R: A recombinant attenuated Salmonella enterica serovar Typhimurium vaccine encoding Eimeria acervulina antigen offers protection against $\mathrm{E}$. acervulina challenge. Infect Immun 2006, 74:6785-6796.

5. McMahon-Pratt $\mathrm{D}$, Rodriguez $\mathrm{D}$, Rodriguez $\mathrm{JR}$, Zhang $\mathrm{Y}$, Manson $\mathrm{K}$ Bergman C, Rivas L, Rodriguez JF, Lohman KL, Ruddle NH, et al: Recombinant vaccinia viruses expressing GP46/M-2 protect against Leishmania infection. Infect Immun 1993, 61:3351-3359.

6. Moss B: Vaccinia virus: a tool for research and vaccine development. Science 1991, 252:1662-1667.

7. Gunilla B, Karlsson PL: Vaccine Protocols.Edited by: Robinson AMJH, Cranage MP. Humana Press; , 2.
8. Deeks SL, Clark M, Scheifele DW, Law BJ, Dawar M, Ahmadipour N, Walop W, Ellis CE, King A: Serious adverse events associated with bacille Calmette-Guerin vaccine in Canada. Pediatr Infect Dis J 2005, 24:538-541.

9. Denkers EY, Gazzinelli RT: Regulation and function of T-cell-mediated immunity during Toxoplasma gondii infection. Clin Microbiol Rev 1998 11:569-588.

10. Abu-Madi MA, Al-Molawi N, Behnke JM: Seroprevalence and epidemiological correlates of Toxoplasma gondii infections among patients referred for hospital-based serological testing in Doha, Qatar. Parasit Vectors 2008, 1:39.

11. Zhao GH, Zhang MT, Lei LH, Shang CC, Cao DY, Tian TT, Li J, Xu JY, Yao YL, Chen DK, Zhu XQ: Seroprevalence of Toxoplasma gondii infection in dairy goats in Shaanxi Province, Northwestern China. Parasit Vectors 4:47.

12. Alvarado-Esquivel C, Estrada-Martinez S: Toxoplasma gondii infection and abdominal hernia: evidence of a new association. Parasit Vectors 4:112.

13. Bhopale GM: Development of a vaccine for toxoplasmosis: current status. Microbes Infect 2003, 5:457-462.

14. Wilkins MF, O'Connell E, Te Punga WA: Toxoplasmosis in sheep III. Further evaluation of the ability of a live Toxoplasma gondii vaccine to prevent lamb losses and reduce congenital infection following experimental oral challenge. $N Z$ Vet J 1988, 36:86-89.

15. Buxton $D$, Innes EA: A commercial vaccine for ovine toxoplasmosis. Parasitology 1995, 110(Suppl):S11-16.

16. Dubey JP: Toxoplasmosis in sheep-the last 20 years. Vet Parasitol 2009, 163:1-14.

17. Meissner M, Breinich MS, Gilson PR, Crabb BS: Molecular genetic tools in Toxoplasma and Plasmodium: achievements and future needs. Curr Opin Microbiol 2007, 10:349-356.

18. Ramirez JR, Gilchrist K, Robledo S, Sepulveda JC, Moll H, Soldati D, Berberich C: Attenuated Toxoplasma gondii ts-4 mutants engineered to express the Leishmania antigen KMP-11 elicit a specific immune response in BALB/c mice. Vaccine 2001, 20:455-461.

19. Denkers EY: T lymphocyte-dependent effector mechanisms of immunity to Toxoplasma gondii. Microbes Infect 1999, 1:699-708.

20. Choi I, Chung HK, Ramu S, Lee HN, Kim KE, Lee S, Yoo J, Choi D, Lee YS, Aguilar B, Hong YK: Visualization of lymphatic vessels by Prox1-promoter directed GFP reporter in a bacterial artificial chromosome-based transgenic mouse. Blood.

21. Hu K, Roos DS, Murray JM: A novel polymer of tubulin forms the conoid of Toxoplasma gondii. J Cell Biol 2002, 156:1039-1050.

22. Hu K, Mann T, Striepen B, Beckers CJ, Roos DS, Murray JM: Daughter cell assembly in the protozoan parasite Toxoplasma gondii. Mol Biol Cell 2002, 13:593-606.

23. Medina-Bolivar F, Wright R, Funk V, Sentz D, Barroso L, Wilkins TD, Petri W $\mathrm{Jr}$, Cramer CL: A non-toxic lectin for antigen delivery of plant-based mucosal vaccines. Vaccine 2003, 21:997-1005.

24. Gubbels MJ, Li C, Striepen B: High-throughput growth assay for Toxoplasma gondii using yellow fluorescent protein. Antimicrob Agents Chemother 2003, 47:309-316.

25. Xiaoxi Huang JZ, Xianyong Liu, Xun Suo: Transgenic Eimeria tenella Expressing Enhanced Yellow Fluorescent Protein (EYFP) as a Model Antigen Stimulated Host Immune Responses to the Heterogenous Protein-Targeted to Different Cellular Compartments.

26. Long PL, Millard BJ, Joyner LP, Norton CC: A guide to laboratory techniques used in the study and diagnosis of avian coccidiosis. Folia Vet Lat 1976, 6:201-217.

27. Hao L, Liu X, Zhou X, Li J, Suo X: Transient transfection of Eimeria tenella using yellow or red fluorescent protein as a marker. Mol Biochem Parasitol 2007, 153:213-215.

28. Roos DS, Donald RG, Morrissette NS, Moulton AL: Molecular tools for genetic dissection of the protozoan parasite Toxoplasma gondii. Methods Cell Biol 1994, 45:27-63.

29. Black M, Seeber F, Soldati D, Kim K, Boothroyd JC: Restriction enzymemediated integration elevates transformation frequency and enables co-transfection of Toxoplasma gondii. Mol Biochem Parasitol 1995, 74:55-63.

30. Zou J, Liu X, Shi T, Huang X, Wang H, Hao L, Yin G, Suo X: Transfection of Eimeria and Toxoplasma using heterologous regulatory sequences. Int $J$ Parasitol 2009, 39:1189-1193.

31. Yun $\mathrm{CH}$, Lillehoj HS, Lillehoj EP: Intestinal immune responses to coccidiosis. Dev Comp Immunol 2000, 24:303-324. 
32. Lillehoj HS: Role of T lymphocytes and cytokines in coccidiosis. Int $J$ Parasitol 1998, 28:1071-1081.

33. Shen H, Miller JF, Fan X, Kolwyck D, Ahmed R, Harty JT: Compartmentalization of bacterial antigens: differential effects on priming of CD8 T cells and protective immunity. Cell 1998, 92:535-545.

34. Zhang G, Huang X, Boldbaatar D, Battur B, Battsetseg B, Zhang H, Yu L, Li Y, Luo $Y$, Cao $S$, et al: Construction of Neospora caninum stably expressing TgSAG1 and evaluation of its protective effects against Toxoplasma gondii infection in mice. Vaccine 28:7243-7247.

35. Shirafuji H, Xuan X, Kimata I, Takashima Y, Fukumoto S, Otsuka H, Nagasawa H, Suzuki H: Expression of P23 of Cryptosporidium parvum in Toxoplasma gondii and evaluation of its protective effects. J Parasitol 2005, 91:476-479.

36. Nishikawa Y, Xuenan X, Makala L, Vielemeyer O, Joiner KA, Nagasawa H: Characterisation of Toxoplasma gondii engineered to express mouse interferon-gamma. Int J Parasitol 2003, 33:1525-1535.

37. O'Connor RM, Kim K, Khan F, Ward HD: Expression of Cpgp40/15 in Toxoplasma gondii: a surrogate system for the study of Cryptosporidium glycoprotein antigens. Infect Immun 2003, 71:6027-6034.

38. O'Connor RM, Wanyiri JW, Wojczyk BS, Kim K, Ward H: Stable expression of Cryptosporidium parvum glycoprotein gp40/15 in Toxoplasma gondii. Mol Biochem Parasitol 2007, 152:149-158.

39. Shirley MW, Ivens A, Gruber A, Madeira AM, Wan KL, Dear PH, Tomley FM: The Eimeria genome projects: a sequence of events. Trends Parasitol 2004, 20:199-201.

40. Kissinger JC, Gajiia B, Li L, Paulsen IT, Roos DS: ToxoDB: accessing the Toxoplasma gondii genome. Nucleic Acids Res 2003, 31:234-236.

41. Kaneto CN, Costa AJ, Paulillo AC, Moraes FR, Murakami TO, Meireles MV: Experimental toxoplasmosis in broiler chicks. Vet Parasitol 1997, 69:203-210.

42. Quan JH, Hassan HA, Cha GH, Shin DW, Lee YH: Antigenemia and specific $\lg \mathrm{M}$ and $\lg \mathrm{G}$ antibody responses in rabbits infected with Toxoplasma gondii. Korean J Parasitol 2009, 47:409-412.

43. Ravindran S, Boothroyd JC: Secretion of proteins into host cells by Apicomplexan parasites. Traffic 2008, 9:647-656.

44. Frickel EM, Sahoo N, Hopp J, Gubbels MJ, Craver MP, Knoll L, Ploegh HL, Grotenbreg GM: Parasite stage-specific recognition of endogenous Toxoplasma gondii-derived CD8+ T cell epitopes. J Infect Dis 2008, 198:1625-1633.

45. Blanchard N, Gonzalez F, Schaeffer M, Joncker NT, Cheng T, Shastri AJ, Robey EA, Shastri N: Immunodominant, protective response to the parasite Toxoplasma gondii requires antigen processing in the endoplasmic reticulum. Nat Immunol 2008, 9:937-944.

46. Kwok LY, Lutjen S, Soltek S, Soldati D, Busch D, Deckert M, Schluter D: The induction and kinetics of antigen-specific CD8 T cells are defined by the stage specificity and compartmentalization of the antigen in murine toxoplasmosis. J Immunol 2003, 170:1949-1957.

47. Wallny HJ, Avila D, Hunt LG, Powell TJ, Riegert P, Salomonsen J, Skjodt K, Vainio O, Vilbois F, Wiles MV, Kaufman J: Peptide motifs of the single dominantly expressed class I molecule explain the striking MHCdetermined response to Rous sarcoma virus in chickens. Proc Natl Acad Sci USA 2006, 103:1434-1439.

doi:10.1186/1756-3305-4-168

Cite this article as: Zou et al.: Evaluation of Toxoplasma gondii as a live vaccine vector in susceptible and resistant hosts. Parasites \& Vectors 2011 4:168.

\section{Submit your next manuscript to BioMed Central and take full advantage of:}

- Convenient online submission

- Thorough peer review

- No space constraints or color figure charges

- Immediate publication on acceptance

- Inclusion in PubMed, CAS, Scopus and Google Scholar

- Research which is freely available for redistribution 